\title{
Grand Challenges for the Study of Cultural Evolution
}

J. Brewer, ${ }^{1}$ M. Gelfand, ${ }^{2}$ J.C. Jackson, ${ }^{3}$ I.F. MacDonald, ${ }^{4}$ P.N. Peregrine, ${ }^{5,6^{*}}$ P.J. Richerson, ${ }^{7}$ P. Turchin, ${ }^{8}$ H. Whitehouse, ${ }^{9}$ D.S. Wilson ${ }^{4}$

${ }^{1}$ Cognitive Policy Works, Seattle, WA 98122, USA. ${ }^{2}$ University of Maryland, College Park, MD 20742, USA. ${ }^{3}$ University of North Carolina, Chapel Hill, NC 27599, USA. ${ }^{4}$ Binghamton University, Binghamton, NY 13902, USA. ${ }^{5}$ Lawrence University, Appleton, WI 54911 USA. ${ }^{6}$ Santa Fe Institute, Santa Fe, NM 87501. ${ }^{7}$ University of California, Davis, CA 95616, USA. ${ }^{8}$ University of Connecticut, Storrs, CT 06269, USA. ${ }^{9}$ Oxford University, Oxford, OX1 4AU, UK. *Corresponding author. E-mail: peter.n.peregrine@lawrence.edu.

A new Cultural Evolution Society (CES) was launched in June 2015 and its founding members were surveyed to identify the major scientific questions and "Grand Challenges" currently facing the study of cultural evolution. We present the results of that survey and discuss their implications for an emergent synthesis in the study of culture based on Darwinian principles. 
The scientific study of culture is currently undergoing a theoretical synthesis comparable to the ongoing synthesis of biological knowledge that began in the $20^{\text {th }}$ century. Critical to both syntheses is the application of Darwinian evolutionary concepts and methods (1). Much like genes, many elements of culture (e.g. technology, language, religion) appear to change through descent with modification (2), and in many cases, genetic and cultural evolution interact in both developmental and evolutionary time (3). In the future, the statement "Nothing about humanity makes sense except in the light of evolution" could be taken for granted in the same way that Dobzhanski's statement "Nothing in biology makes sense except in the light of evolution" is taken for granted today.

Progress toward a $21^{\text {st }}$ century synthesis in the study of cultural evolution has been slow. Darwin's original effort to incorporate humans into the theory of evolution in the Descent of Man (4) was sophisticated but his treatment was neglected by most social scientists of the early $20^{\text {th }}$ century. The publication of E.O. Wilson's Sociobiology: The New Synthesis (5) was hailed as a triumph in the biological sciences - but the final chapter including humans in the new synthesis was met with widespread resistance from social scientists and some evolutionary biologists (6). At the time it was unacceptable to most social scientists to study human social behavior and cultural diversity from an evolutionary perspective. Yet within a decade, terms such as "Evolutionary Psychology”, "Evolutionary Anthropology”, "Evolutionary Medicine”, "Gene-Culture Coevolution," "Evolutionary Economics", "Evolutionary Religious Studies”, “Literary Darwinism”, and "Universal Darwinism” emerged, signaling an attempt to rethink the human sciences from a modern evolutionary perspective. These new schools of thought were controversial — and embryonic — but nevertheless began to prove themselves on the playing field of science in the form of peer-reviewed books and articles. Yet despite most major topics within 
the human sciences and humanities now being considered from an evolutionary perspective (7), much of the explanatory potential of the cultural evolution field remains as yet unfulfilled (8). Only a small fraction of the worldwide academic community comfortably use the evolutionary toolkit, and this scarcity also translates to the general public and countless people and institutions that are trying to accomplish positive cultural change in a practical sense.

\section{A new society}

To catalyze a theoretical synthesis in the study of culture it is vital to hasten the acceptance of evolutionary thinking across the humanities and human sciences. A new Cultural Evolution Society (CES) was launched in June 2015 in order to foster this process. Over 1600 people representing more than 30 disciplines and from over 50 nations expressed an interest in CES and over 600 have become founding members, clearly indicating the desire for such a society. As a seedbed group, CES organizers wanted to expand our understanding of the status of current cultural evolutionary scholarship and to identify the major scientific questions that motivate the CES membership by reaching out to all the founding members to ask what they regarded as the "Grand Challenges" facing the field today.

A total of 236 CES members from around the world completed an online questionnaire in which they could nominate challenges and provide a brief description and rationale for each. A total of 422 Grand Challenge Ideas (GCIs) were received. These GCIs were analyzed using close-text semantic analysis, in which each text entry was carefully read and coded for thematic content (9). Content topics were intentionally defined loosely and in a manner that allowed clustering of responses based on emerging themes, with each entry typically receiving several codes to cover the full range of ideas expressed. The top five thematic codes, in terms of 
frequency of overall occurrence, were "knowledge synthesis", "prosociality", "culture definition", "environment" and "cultural transmission", and reflect major themes in the field of cultural evolution.

To further aid in interpretation and get a better sense of the relationships between the identified themes, the co-occurrence network of the top 30 themes was analyzed (see Figure 1). The resultant graph demonstrates extensive interconnections between the themes, although there is evidence of distinct clusters. One cluster (characterized by its largest node "knowledge synthesis" and colored red) contains themes relating primarily to issues of theoretical integration and speaks to the idea that while many behavioral scientists and humanities scholars see culture as a defining feature of humankind, different subfields rarely read each other's work or build interdisciplinary research programs to explore how human cultures differ from those of other animals. A second cluster (“cultural transmission"; green) is largely centered on understanding the dynamics of cultural systems (i.e. the subject matter of the field). Cultures in general, and human cultures in particular, are rich in diversity, and comparing them offers measures of variation in human social behavior that can be used to empirically explore cultural stability and change. A third cluster ("prosociality"; beige) covers topics related to the evolution of large and complex human societies. It also links to themes relating to real-world applications of our understanding of cultural evolutionary processes in order to improve various aspects of the collective human experience ("environment", "economics", governance", etc.). Finally, a smaller cluster ("education"; blue) relates to education and outreach beyond the scientific community. Overall, the density of interconnections means that none of the clusters is independent from the others and suggests that cultural evolution is a coherent field rather than an ad hoc combination of two or more distinctive fields. 
With these interconnections and clusters in mind, the topics identified through the Grand Challenges survey were organized into broader themes that could be approached as either an integrative research agenda or applied intervention. These themes were then pared down to fourteen preliminary Grand Challenges. The preliminary Grand Challenges were circulated among the authors and condensed to the list in Table 1. The Grand Challenges are not listed in any particular order, and certainly not by any assumed priority. While the Grand Challenges do not comprise an exhaustive list of challenges, and are not necessarily shared by all scholars working in the field of cultural evolution, they constitute areas in which research on cultural evolution might focus over the next decade in order to further a theoretical synthesis.

\section{Putting the survey into action}

The impact of these Grand Challenges might be maximized in two ways. The first would be to develop field sites for the study of cultural evolution, similar to those that have long been central to research in evolutionary ecology. These would not be limited to social insects or any particular group of animals, but might cover a range of cultural organisms to examine similarities and differences in cultural adaptations to a given environments, to undertake experimental studies that impact multiple species and their interactions, and perhaps even to explore cross-species transmission of cultural traits. Human field sites might also be developed. While archaeologists have routinely excavated long-term occupation sites that provide a picture of cultural evolution, an important addition would be field sites to explore cultural evolution in modern populations. Several long-term field sites exist but coverage is limited given the scale human cultural diversity. Distributed ethnographic and psychological projects are becoming more common and these could develop into a type of distributed field site that would allow a group of scholars 
working in different locations to coordinate their efforts to understand similarities and differences in cultural adaptations $(11,12)$.

A second way of maximizing the impact of the Grand Challenges would be to develop coordinated research projects involving investigators from multiple disciplines, but focused on one of the Grand Challenge topics or questions. Such coordinated projects are already becoming common but the Grand Challenges could provide a focus so that these projects are coordinated not only internally but across one another, allowing diverse research groups to focus on areas of specific interest while simultaneously contributing to our understanding of broad topics that are integral to furthering synthesis in the study of cultural evolution. Indeed, the Grand Challenges might provide a focus to justify specific projects to funders by illustrating how they contribute to a larger research initiative that is itself tied to a broad attempt at synthesis.

Many responses to the Grand Challenges questionnaire included references to applied work. There seems a great interest among those who study cultural evolution to put their knowledge into practice in order to inform current political debates and to develop interventions producing a more equitable and sustainable world. The Grand Challenges and the synthesis they are intended to catalyze may produce impacts far beyond our current understanding of cultural evolution, extending into mechanisms to improve human life. The $20^{\text {th }}$ century biological synthesis has already accomplished this in previously unimaginable ways: engineering disease and drought resistant food, developing life-saving drugs and therapies, and transforming our understanding of human impact on the environment, among many others. Respondents to the Grand Challenges survey see the $21^{\text {st }}$ century synthesis for the study of cultural evolution as having the same potential to address problems of social inequality, conflict, sustainability, and the continuing anthropogenic restructuring of the earth. 


\section{References:}

1. H. Gintis, Individuality and Entanglement: The Moral and Material Bases of Social Life (Princeton University Press, Princeton, NJ, 2015).

2. P.J. Richerson, M.H. Christiansen, Cultural Evolution: Society, Technology, Language, and Religion (MIT Press, Cambridge, MA, 2013).

3. K.N. Laland, J. Odling-Smee, S. Myles, How culture shaped the human genome: bringing genetics and the human sciences together. Nat. Rev. Gen. 11, 137-148(2010)..

4. C. Darwin, The Descent of Man, and Selection in Relation to Sex (John Murray, London, 1871).

5. E.O. Wilson, Sociobiology: The New Synthesis (Belknap Press of Harvard University, Cambridge, MA, 1975).

6. U. Segerstråle, Defenders of the Truth: The Battle for Science in the Sociobiology Debate and Beyond (Oxford University Press, New York, 2000).

7. T. Lewens, Cultural Evolution (Oxford University Press, UK, 2015).

8. A. Mesoudi, Cultural evolution: A review of theory, findings and controversies. Evol. Biol. (2015) doi:10.1007/s11692-015-9320-0.

9. E. Haas, G. Fischman, Nostalgia, entrepreneurship, and redemption: Understanding prototypes in higher education. Am. Ed. Res. J. 47, 532-562 (2010).

10. M. Bastian, S. Heymann, M. Jacomy, Gephi: An Open Source Software for Exploring and Manipulating Networks. International AAAI Conference on Weblogs and Social Media (2009). 11. D.S. Wilson and H. Whitehouse, Developing the field site concept for the study of cultural evolution. Social Evolution Forum, https://evolution-institute.org/focus-article/developing-thefield-site-concept-for-the-study-of-cultural-evolution-two-essays-by-david-sloan-wilson-andharvey-whitehouse, (Oct. 16, 2016) 
12. R. McElreath, A Long-Form Research Program in Human Behavior, Ecology, and Culture. http://www.eva.mpg.de/fileadmin/content_files/staff/richard_mcelreath/pdf/HBEC_whitepaper.p df, (Oct. 6, 2016) 
Table 1: Grand Challenges for the Study of Cultural Evolution

\begin{tabular}{|c|c|}
\hline $\begin{array}{l}\text { 1. Understanding the Role of } \\
\text { Social Adaptation in Cultural } \\
\text { Evolution. }\end{array}$ & $\begin{array}{l}\text { Culture is often conceived of as a means of adaptation that is not inherent to biological } \\
\text { processes. Such adaptations are usually viewed as technological in nature, but they probably } \\
\text { generalize to many non-technological domains. What, for instance, are the possible adaptive } \\
\text { roles of social structures and behaviors such as sharing, kinship, and capital punishment? What } \\
\text { are the most critical ecological and social conditions that drive social adaptation? What are the } \\
\text { differences (and similarities) between biological and social adaptation? }\end{array}$ \\
\hline $\begin{array}{l}\text { 2. Understanding the Role of } \\
\text { Cultural Evolution in the } \\
\text { Context of Organic Evolution }\end{array}$ & $\begin{array}{l}\text { Recent investigations into gene-culture coevolution show that culture is strongly influenced by } \\
\text { biology, and influences biological processes in turn. Understanding these processes in any } \\
\text { detail is a decades-long challenge made exciting by recent advances in many fields such as } \\
\text { ancient DNA recovery, the comparative analysis of cultures, and the developmental psychology } \\
\text { of culture acquisition. }\end{array}$ \\
\hline $\begin{array}{l}\text { 3: Modeling Culture as a } \\
\text { Complex Adaptive System. }\end{array}$ & $\begin{array}{l}\text { Systems theory has led to profound insights in disciplines from physics to economics. Culture } \\
\text { can easily be understood as a complex adaptive system. Can the application of systems theory } \\
\text { aid us in understanding cultural evolution? For example, can dynamic shifts in organization } \\
\text { such as the development of cities be understood as emergent properties? Do innovations create } \\
\text { feedback loops leading to cumulative culture? Are cultural collapses understandable as } \\
\text { outcomes of non-linear interactions? Do rules exist for "local" interactions at the small scale of } \\
\text { individual agents that give rise to "global" patterns at the social scale? }\end{array}$ \\
\hline $\begin{array}{l}\text { 4. Identifying Processes of } \\
\text { Transmission and } \\
\text { Accumulation of Cultural } \\
\text { Traits. }\end{array}$ & $\begin{array}{l}\text { While there has been important work done on the mathematical modeling of cultural } \\
\text { transmission, there is not yet consensus on the mechanisms underlying those models. What are } \\
\text { the cognitive and behavioral processes underlying cultural transmission? How are innovations } \\
\text { selectively transmitted over existing technologies or behaviors? How do differentiated social } \\
\text { statuses, roles, and educational systems impact cultural transmission? How can we most } \\
\text { usefully conceptualize the units of cultural transmission? What does it mean when we say a } \\
\text { culture evolves? }\end{array}$ \\
\hline $\begin{array}{l}\text { 5: Integrating Methods, Data, } \\
\text { and Results across Disciplines. }\end{array}$ & $\begin{array}{l}\text { Cultural evolutionary research takes place in many different organizations and departments, by } \\
\text { scholars of many different disciplines, and on subjects ranging from literary analysis to } \\
\text { biochemistry. Establishing shared conceptual references and vocabulary is fundamental. } \\
\text { Finding common data structures, common language, common research methods, and common } \\
\text { publication outlets is also essential, and poses an enormous challenge for cultural evolutionary } \\
\text { research. }\end{array}$ \\
\hline
\end{tabular}




\begin{tabular}{|l|l|}
\hline $\begin{array}{l}\text { 6: Creating New Organizational } \\
\text { and Funding Structures that } \\
\text { Support Interdisciplinary } \\
\text { Research and Teaching. }\end{array}$ & $\begin{array}{l}\text { Cultural evolutionary research is, by its very nature, interdisciplinary, but most academic } \\
\text { institutions are not built to foster or even to support truly interdisciplinary work. Funding } \\
\text { agencies tend to promote disciplinary boundaries, only funding within narrow. Higher } \\
\text { education is typically structured around common disciplinary cores and elective courses. } \\
\text { Breaking down the walls separating disciplines and creating institutional, educational, and } \\
\text { research space for interdisciplinary engagement will be a major challenge for research on } \\
\text { cultural evolution. }\end{array}$ \\
\hline $\begin{array}{l}\text { 7. Identifying Cultural } \\
\text { Evolutionary Processes that } \\
\text { Address Significant Social, } \\
\text { Economic, and Political } \\
\text { Problems. }\end{array}$ & $\begin{array}{l}\text { The study of cultural evolution provides empirical evidence for processes that lead to } \\
\text { successful outcomes and those that lead to failures. Applied cultural evolutionary research can } \\
\text { aspire to provide insights into complex problems facing the world today such as poverty, } \\
\text { climate change, terrorism, among many others, and offer potential solutions to those problems. } \\
\text { It should also elucidate the diversity of cultural norms, ideologies, value systems, and } \\
\text { situational ethics at play in policy formulation (especially when working across cultures). }\end{array}$ \\
\hline $\begin{array}{l}\text { 8. Educating Policy Makers and } \\
\text { the Public about Cultural } \\
\text { Evolution. }\end{array}$ & $\begin{array}{l}\text { Providing insights and answers to social, economic, and political problems is not sufficient if } \\
\text { policy makers and the public remain ignorant of those insights and answers. Applications aside, } \\
\text { human and animal cultural evolution is a topic that fascinates the public. An important } \\
\text { challenge for cultural evolutionary research is to establish means of engaging with policy } \\
\text { makers and the public to disseminate the findings of research and to offer expert advice on } \\
\text { significant world problems. }\end{array}$ \\
\hline
\end{tabular}


Figure 1. Frequency and co-occurrences of themes identified in the Grand Challenges survey responses. Node (theme) size is scaled to the number of times each appeared in the pool of Grand Challenge Ideas (GCIs) and edges (co-occurrences) are scaled to the number of times any two themes appeared in a given GCI nomination. Node color indicates cluster membership as revealed by a community detection algorithm applied using open source software (10); clusters are laid out clockwise in decreasing order based on the size of each clusters largest node (i.e. most frequently occurring theme). See article text for interpretation of the clusters.

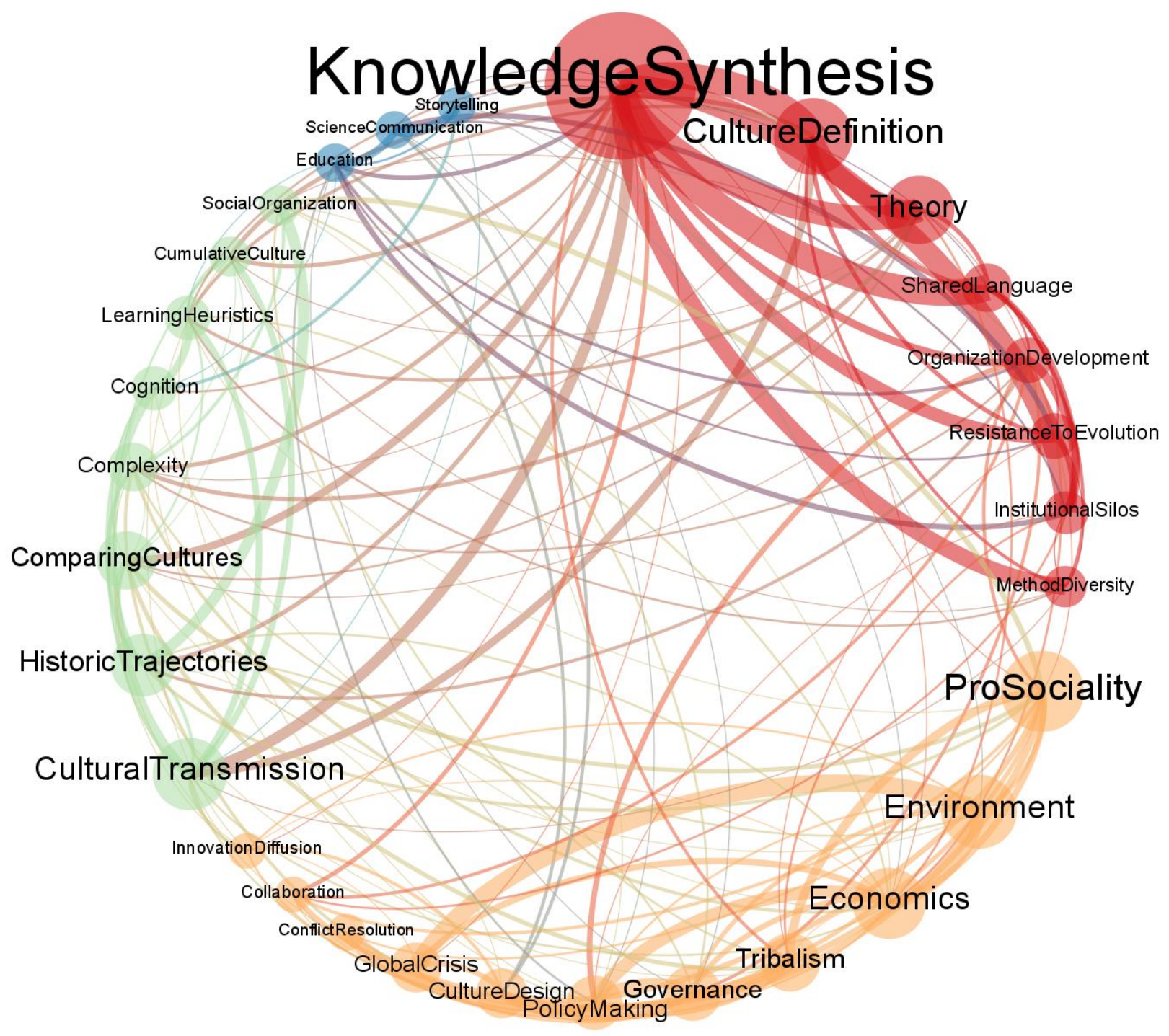


Acknowledgements: The authors wish to thank the Evolution Institute and the University of Maryland for assisting with arrangements and providing space for the meetings in which the Cultural Evolution Society was organized and the Grand Challenges survey was developed. These organizational meetings and the analysis of the Grand Challenges survey results were funded through a grant to the Evolution Institute from the John Templeton Foundation. We also wish to thank the anonymous reviewers who aided us in improving this Comment.

Author Contributions: Brewer and MacDonald conducted the "Grand Challenges Survey" and processed the initial results. Peregrine analyzed the processed results to draft the preliminary list of "Grand Challenges." All the authors evaluated the initial list of "Grand Challenges" and aided in parsing them to the final eight. Brewer, MacDonald, Peregrine, and Wilson wrote the initial draft of the manuscript, and all the authors contributed to revisions.

Reprints and permissions information is available at www.nature.com/reprints.

The authors have no competing financial interests.

Correspondence and requests for materials should be addressed to peter.n.peregrine@lawrence.edu 